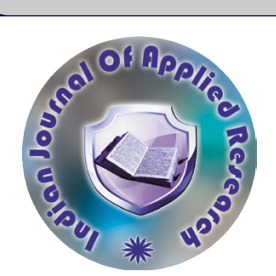

Radiodiagnosis

\title{
USEFULNESS OF VARIOUS DYNAMIC AND STATIC PARAMETERS IN SLEEP MRI STUDIES - DO THEIR INCORPORATION IN ROUTINE REPORTING IN PATIENTS WITH OBSTRUCTIVE SLEEP APNEA PROVE VALUABLE?
}

\section{Amarnath Chellathurai}

Sudhakar Vadivel

\section{Chezhian} Jayabalan*

\section{Priya Muthaiyan}

Sebastian Xavier
Professor and Head of the Department,Department of Radio-Diagnosis, Government Stanley Medical College, Chennai-600001

Associate Professor,Department of Radio-Diagnosis, Government Stanley Medical College, Chennai-600001

Associate Professor,Department of Radio-Diagnosis, Government Stanley Medical College, Chennai-600001 *Corresponding Author

Assistant Professor,Department of Radio-Diagnosis, Government Stanley Medical College, Chennai-600001

Junior Resident,Department of Radio-Diagnosis, Government Stanley Medical College, Chennai-600001

ABSTRACT Background:Sleep MRI is a new emerging non invasive modality in the preoperative evaluation of patients with obstructive sleep apnoea. Rather than subjective, the aim of the study is to evaluate the competency of certain dynamic and static parameters (variables and diemensions), that can be used in day to day reporting. Incorporating such parameters, may prove to be helpful in guiding surgical decision making.

Materials and Methods:A retrospective analytical study included 27 subjects with OSA aged between 18 and 70 years using $1.5 \mathrm{~T}$ MRI . We measured variables such as the cross-sectional area and diameter of the retropalatal and retroglossal airway during the asleep and awakened states.We also measured comprehensive airway dimensions such as antero-posterior diameter of the tongue, midline saggital diameter of tongue, intermandibular rami distance, mentum spine distance and relative size of the tongue using the spine as the posterior border of the bony confines.

Results:21 patients had significant retropalatal airway compromise, and was evidenced by statistically significant variables in awakened and asleep states. The mean minimum anterior-posterior diameter $(\mathrm{mm})$ of retropalatal airway during respiratory cycle was statistically significant measuring $3.1 \pm 1.5 \mathrm{~mm}$ in awaken state and $1.1 \pm 1.5 \mathrm{~mm}$ during asleep state (p- value 0.01 ). The mean minimum cross sectional area during respiratory cycle was statistically significant measuring $98 \pm 11 \mathrm{~mm} 2$ in awaken state and $63 \pm 12 \mathrm{~mm} 2$ during asleep state (p- value 0.04$)$. 6 patients had significant retroglossal airway compromise, and was evidenced by statistically significant. The thickness of the soft palate and the relative size of the tongue $(0.5 \pm 0.01 \mathrm{~mm}$ ) was statistically significant in these patients ( $\mathrm{p}$ - value 0.01 ).

Conclusion:Our study shows that retropalatal airway is a significant cause of obstruction in patients with OSA, compared with retroglossal airway. We suggest that, when the compromise of the retroplatine aiway is to be evaluated, variables such as cross sectional area and anteroposterior diameter of the retropalatal airway during the respiratory phase in asleep and awakened states proves valuable and dimensions such as soft palate thickness as tool for objective measurement of OSA. When the compromise of the retroglossal aiway is to be evaluated, the relative size of the tongue, proves offers more advantage than the variables of airway compromise such as area and antero-posterior diameter.To further evaluate if some of our parameters correlate with the outcomes of the surgeries of OSA, a multivariate analysis would have been required.

KEYWORDS : Obstructive Sleep Apnoea; Sleep MRI; retroglossal airway ; retropalatal airway ; relative size of the tongue.

\section{INTRODUCTION:}

OSA(Obstructive Sleep Apnea) is characterized by repetitive partial or complete upper airway collapse during sleep, resulting in disrupted normal sleep architecture and associated with arterial desaturations. The diagnosis of obstructive sleep apnoea is confirmed by overnight polysomnography (PSG) ${ }^{1}$. Sleep MRI is a new emerging non invasive modality in the preoperative evaluation of patients with obstructive sleep apnea. MR sleep studies are used to identify as close as to the (patterns of collapse) causes of OSA, such as recurrent and enlarged adenoid tonsils, enlarged lingual tonsils, glossoptosis, hypopharyngeal collapse, or an enlarged soft palate. Rather than subjective, the aim of our study is to evaluate the competency of certain dynamic and static parameters (referred to as variables and dimensions respectively), that can be used in day to day reporting. Incorporating such parameters, may prove to be helpful in guiding surgical decision making.

The incidence of OSA is on the rise on par with the global epidemic of obesity and with it also increases the occurrence and probability of the comorbid conditions such as coronary artery disease, diabetes, syndrome X, hypertension, and thus impaired daily life activities and sometimes marital discordance ${ }^{2}$. "Obstructive sleep apnea (OSA)" and "Upper Airway Resistance Syndrome (UARS)" are two distinct and related terms in the spectrum of Sleep-Disordered Breathing $(\mathrm{SDB})^{3,4}$.If the respiratory events occur $>5$ times per hour of sleep and associated with symptoms, most commonly snoring, excessive daytime fatiguability, and witnessed apneas , the term obstructive sleep apnea/hypopnea syndrome(OSAHS) is used ${ }^{5}$.

\section{MATERIALSAND METHODS:}

\section{Study Design:}

A retrospective analytical study 36 subjects recruited from, evaluated, and followed by the Department of Otolaryngology-Head and Neck Surgery, Stanley Medical College, Chennai . The study protocol was approved by the Institutional Ethical Committee and written informed consent was obtained from all subjects.

\section{Subjects:}

Inclusion criteria were (1) 18 to 70 years of age; (2) No evidence of claustrophobia; (3) Evidence of sleepiness or functional abnormality as determined by Epworth Sleepiness Score (ESS) and Functional Outcomes Sleepiness Questionnaire (FOSQ) in OSA subjects; (4) AHI $>15$ events/hour for OSA subjects. (5) We excluded patients who were pregnant, those with a body mass index (BMI) greater than $40 \mathrm{~kg} / \mathrm{m}^{2}$ (restrictions imposed by the magnetic bore) and those with other contraindications to $\mathrm{MRI}^{6}$.

\section{MRI Protocols :}

MR sleep studies were performed on a 1.5-T MRI unit (Siemens Magnetom Aera, Germany) with the patients positioned supine and in a head and neck vascular coil or a cervical spine coil. Imaging was done during both asleep and awaken states. A screening brain FLAIR axial- brain MRI was done to rule out any structural abnormalities. Neither positive pressure nor artificial airways were used during image 
acquisition. Imaging sequences used in the study included sagittal and axial fast spin-echo inversion-recovery (sagittal turbo IR/TIRM ), sagittal and axial proton density, and sagittal gradient-echo cine.

The technique used in cine MR imaging where 128 consecutive images were done over 2 minutes during the episodes of airway obstruction oxygen desaturation, each image approx 1 second. The images done within second of each other, can be presented in a cine movie format. Images acquired were in two sets, one awake and another asleep.

The technical parameters for the sagittal turbo IR/TIRM sequences were as follows: TR/TE, 5,000/34; inversion time (TI), 155 milliseconds; bandwidth, $15.63 \mathrm{MHz}$; slice thickness, $4 \mathrm{~mm}$; gap, 1 $\mathrm{mm}$; matrix, $256 \times 192$; field of view (FOV), $22 \mathrm{~cm}$; echo-train length, 12 ; and number of excitations, 2 .

The technical parameters for the axial turbo IR/TIRM sequences were 5,000/34; TI, 155 milliseconds; bandwidth, $15.63 \mathrm{MHz}$; slice thickness, $6 \mathrm{~mm}$; gap, $2 \mathrm{~mm}$; matrix, $256 \times 192$; FOV, $22 \mathrm{~cm}$; echotrain length, 12; and number of excitations, 2 . The technical parameters for the sagittal proton density sequences without fat saturation were a TR of 4,000 milliseconds; TE, minimum full; slice thickness, $3 \mathrm{~mm}$; gap, $0.5 \mathrm{~mm}$; matrix, $256 \times 192$; FOV, $24 \mathrm{~cm}$; echo-train length, 12; bandwidth, $15.63 \mathrm{MHz}$; and number of excitations, 2 .

The technical parameters for the axial proton density sequences without fat saturation were 4,000/minimum full; slice thickness, 3 $\mathrm{mm}$; gap, $0.5 \mathrm{~mm}$; matrix, $256 \times 192$; FOV, $24 \mathrm{~cm}$; echo-train length, 12; bandwidth, $20.83 \mathrm{MHz}$; and number of excitations, 2.

The technical parameters for the sagittal gradient- echo cine sequence were TE, minimum full; TI, 25 milliseconds; bandwidth, $31.25 \mathrm{MHz}$; slice thickness, $12 \mathrm{~mm}$; matrix, $256 \times 128$; FOV, $22 \mathrm{~cm}$; and number of excitations, 2

\section{MRI Dimensions and Variables :}

Dynamic Parameters of the Retropalatal and Retroglossal airway (Variables):

The cross-sectional area of the retropalatal airway (RPA) was measured between the nasal and occlusal planes (distance between the soft palate to the posterior pharyngeal wall) were determined on the sagittal images both at the time of maximum and minimum cross- way between the nasal and occlusal planes on the sagittal images both at the time of maximum and minimum cross-sectional areas over time (Fig 1.A\&B). The presence or absence of collapse of the nasopharynx was also noted 7-9.

Figure 1. (A) The maximum diameter of the retropalatal airway was measured in the mid-way between the nasal and occlusal planes (distance between the soft palate to the posterior pharyngeal wall) on the sagittal images during awakened state. 1 (B) The minimum diameter of the retropalatal airway was measured in the mid-way between the nasal and occlusal planes (distance between the soft palate to the posterior pharyngeal wall) on the sagittal images during awakened state.
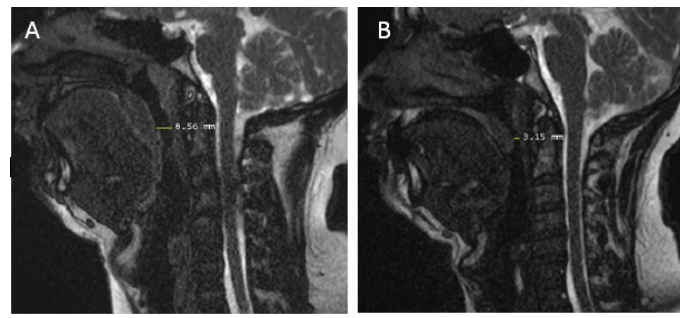

The cross-sectional area and AP diameter of the retroglossal airway (RGA) between the inferior aspect of the soft palate and the superior aspect of the epiglottis were determined on the sagittal images both at the time of maximum and minimum cross-sectional areas over time. The presence or absence of collapse of the orophayngeal airway was also noted ${ }^{10}$

\section{Static Parameters of the Retropalatal and Retroglossal airway} (Dimensions):

The thickness of the soft palate was measured at its greatest dimension. The maximum AP diameter of the tongue was made by drawing a line from the posterior cortex of the mental portion of the mandible to the most posterior aspect of the tongue base. Two measurements of the bony confines of the pharynx were measured on proton density-weighted images without fat saturation: intermandibular rami distance and mentum - spine distance. The intermandibular rami distance was measured on axial images from the inner cortex of each mandibular ramus at the posterior aspect of the ramus near the angle of the mandible (Fig 2A\&B). The mentum-spine distance was measured between the posterior cortex of the mandibular mentum to the anterior cortex of the $\mathrm{C} 2$ vertebral body ${ }^{11-13}$.

Figure 2. (A) The intermandibular rami distance measured on axial images from the inner cortex of each mandibular ramus. (B)The mentum-spine distance measured between the posterior cortex of the mandibular mentum to the anterior cortex of the $\mathrm{C} 2$ vertebral body

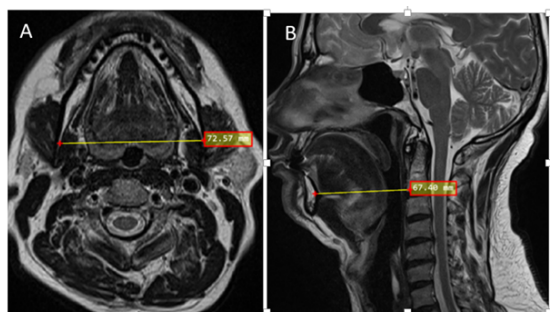

The cross-sectional area of the tongue at the midsagittal sections were obtained by drawing a region of interest around the tongue borders (Fig $3 \mathrm{~A})$. From these measurements, a comprehensive ratio is calculated as follows: the relative size of the tongue using the spine as the posterior border of the bony confines $=$ [midline sagittal tongue area/ (intermandibular rami distance $\times$ mentum-spine distance) $]^{14}$.

Figure 3 : (A) The cross sectional area of the tongue measured in sagittal T2 W- MRI. (B)The cross sectional of the retropalatal airway was measured in the mid-way between the nasal and occlusal planes (distance between the soft palate to the posterior pharyngeal wall) on the sagittal images during asleep state

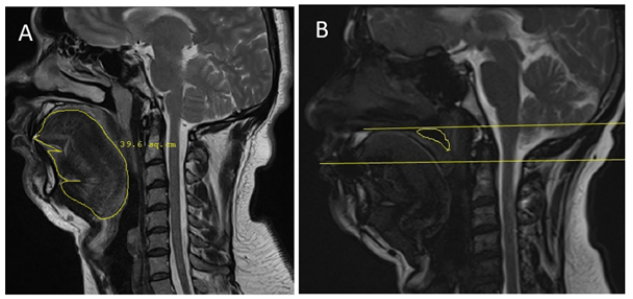

\section{RESULTS:}

We evaluated the dynamic MRI parameters of 27 patients with OSA syndrome aged between 18 and 70 years. The gender ratio was slightly asymmetrical but not statistically different (p-value 0.68 ), with 9 female patients and 18 male patients. Further, OSA subjects reported various levels of functional disturbance and we did not make a correlate between the clinical severity with MRI findings. The term "variable or dynamic parameter" used in this context, is defined as " change in measurements during the respiratory cycle" eg. Retroglossal airway cross sectional area. The term "dimension or static parameter" used in this context, is defined as "no change in measurement during respiratory cycle". eg. surface area of tongue. The term "parameter" used in this context, is either a variable or dimension or both $\mathrm{h}^{15}$

Table 1: Dynamic parameters of Retropalatal airway during awakened and asleep states.

\begin{tabular}{|l|l|l|l|l|}
\hline Variables of the retropalatal airway: $(\boldsymbol{n}=\mathbf{2 1})$ \\
\hline S.No & Variables & Awake & Asleep & p-value \\
\hline 1 & $\begin{array}{l}\text { Minimum anterior-posterior } \\
\text { diameter (mm) of retropalatal airway } \\
\text { during respiratory cycle }\end{array}$ & $3.1 \pm 1.5$ & $1.1 \pm 1.5$ & 0.01 \\
\hline 2 & $\begin{array}{l}\text { Maximum anterior-posterior } \\
\text { diameter (mm) of retropalatal airway } \\
\text { during respiratory cycle }\end{array}$ & $5.1 \pm 2.0$ & $4.9 \pm 2.0$ & 0.09 \\
\hline 3 & $\begin{array}{l}\text { Maximum area of retropalatal airway } \\
\text { during respiratory cycle }\left(\mathrm{mm}^{2}\right)\end{array}$ & $173 \pm 16$ & $158 \pm 12$ & 0.06 \\
\hline 4 & $\begin{array}{l}\text { Minimum area of retropalatal airway } \\
\text { during respiratory cycle }\left(\mathrm{mm}^{2}\right)\end{array}$ & $98 \pm 12$ & $63 \pm 11$ & 0.04 \\
\hline
\end{tabular}


Table 2: Dynamic parameters of Retropalatal airway during awakened and asleep states.

\begin{tabular}{|c|c|c|c|c|}
\hline \multicolumn{5}{|c|}{ Variables of the retropalatal airway: $(n=6)$} \\
\hline S.No & \begin{tabular}{|l|} 
Variables \\
\end{tabular} & Awake & Asleep & p-value \\
\hline 6 & $\begin{array}{l}\text { Minimum anterior-posterior } \\
\text { diameter }(\mathrm{mm}) \text { of retropalatal airway } \\
\text { during respiratory cycle }\end{array}$ & $3.1 \pm 1.5$ & $2.9 \pm 1.2$ & 0.18 \\
\hline 7 & $\begin{array}{l}\text { Maximum anterior-posterior } \\
\text { diameter }(\mathrm{mm}) \text { of retropalatal airway } \\
\text { during respiratory cycle }\end{array}$ & $5.1 \pm 2.0$ & $4.9 \pm 2.0$ & 0.09 \\
\hline 8 & $\begin{array}{l}\text { Maximum area of retropalatal } \\
\text { airway during respiratory } \\
\text { cycle }\left(\mathrm{mm}^{2}\right)\end{array}$ & $172 \pm 16$ & $167 \pm 12$ & 0.06 \\
\hline 9 & $\begin{array}{l}\text { Minimum area of retropalatal airway } \\
\text { during respiratory cycle }\left(\mathrm{mm}^{2}\right)\end{array}$ & $97 \pm 12$ & $91 \pm 11$ & 0.14 \\
\hline
\end{tabular}

Table 3: Static parameters of Retropalatal airway.

Dimensions of the retro palatal airway $(n=21)$

\begin{tabular}{|l|l|l|l|l|}
\hline S.No & Diemensions & $\begin{array}{l}\text { OSA } \\
\text { cases }\end{array}$ & $\begin{array}{l}\text { Controls from } \\
\text { various studies }\end{array}$ & $\begin{array}{l}\text { p- } \\
\text { value }\end{array}$ \\
\hline 5 & Soft palate thickness $(\mathrm{mm})$ & $11.2 \pm 0.38$ & $8.9 \pm 0.51$ & 0.04 \\
\hline
\end{tabular}

Table 4: Dynamic parameters of Retroglossal airway during awakened and asleep states.

\begin{tabular}{|l|l|l|l|l|}
\hline \multicolumn{6}{|l|}{ Variables of the retroglossal airway: $(\boldsymbol{n}=\mathbf{6})$} \\
\hline S.No & Variables & Awake & Asleep & p-value \\
\hline 10 & $\begin{array}{l}\text { Minimum anterior-posterior } \\
\text { diameter (mm) of retroglossal } \\
\text { airway during respiratory cycle }\end{array}$ & $1.0 \pm 0.2$ & $0.8 \pm 0.4$ & 0.49 \\
\hline 11 & $\begin{array}{l}\text { Maximum anterior-posterior } \\
\text { diameter (mm) of retroglossal } \\
\text { airway during respiratory cycle }\end{array}$ & $4.8 \pm 0.4$ & $4.7 \pm 0.6$ & 0.81 \\
\hline 12 & $\begin{array}{l}\text { Minimum area of retroglossal } \\
\text { airway during respiratory cycle } \\
\left(\text { mm }^{2}\right)\end{array}$ & $54.9 \pm 8$ & $46.6 \pm 12$ & 0.42 \\
\hline 13 & $\begin{array}{l}\text { Maximum area of retroglossal } \\
\text { airway during respiratory cycle } \\
\left(\mathrm{mm}^{2}\right)\end{array}$ & $163.9 \pm 17$ & $160.2 \pm$ & 0.08 \\
\hline
\end{tabular}

Table 5: Static parameters of Retroglossal airway.

Dimensions of the retroglossal airway $(n=6)$

\begin{tabular}{|l|l|l|l|l|}
\hline S.No & Diemensions & OSA cases & Controls* & p-value \\
\hline 14 & Tongue- Midline AP $(\mathrm{mm})$ & $45 \pm 1.5$ & $44.2 \pm 1.3$ & 0.27 \\
\hline 15 & $\begin{array}{l}\text { Midline sagittal tongue area } \\
\left(\mathrm{mm}^{2}\right)\end{array}$ & $31.2 \pm 3.5$ & $30.1 \pm 3.5$ & 0.65 \\
\hline 16 & $\begin{array}{l}\text { Intermandibular rami } \\
\text { distance (mm) }\end{array}$ & $74.2 \pm 1$ & $74.6 \pm 1.3$ & 0.66 \\
\hline 17 & Mentum-spine distance $(\mathrm{mm})$ & $61.1 \pm 1.9$ & $58.3 \pm 2.0$ & 0.06 \\
\hline 18 & $\begin{array}{l}\text { Relative size of tongue using } \\
\text { spine as border }\end{array}$ & $0.5 \pm 0.01$ & $\begin{array}{l}0.46 \pm \\
0.02\end{array}$ & 0.05 \\
\hline
\end{tabular}

*The controls used in the study are from patients with significant retropalatal airway obstruction alone.

We classified OSAS subjects into the following: subjects with retropalatal airway compromise and those with retroglossal airway compromise. Firstly, dynamic parameters of retropharyngeal airway were evaluated for all patients during awakened and asleep states. For 6 patients who did not reveal significant difference in these parameters , were subjected to measurements of dynamic and static retroglossal airway parameters

21 patients had significant retropalatal airway compromise, and was evidenced by statistically significant variables in awakened and asleep states (Table 1). The mean minimum anterior-posterior diameter (mm) of retropalatal airway during respiratory cycle was statistically significant measuring $3.1 \pm 1.5 \mathrm{~mm}$ in awaken state and $1.1 \pm 1.5 \mathrm{~mm}$ during asleep state (p-value 0.01 ). The mean minimum cross sectional area $\left(\mathrm{mm}^{2}\right)$ of retropalatal airway during respiratory cycle was statistically significant measuring $98 \pm 12 \mathrm{~mm}^{2}$ in awaken state and 63 $\pm 11 \mathrm{~mm}^{2}$ during asleep state ( $\mathrm{p}$ - value 0.04 ).

6 patients who did not reveal significant difference in these retropalatal parameters, were subjected to measurements of dynamic and static retroglossal airway parameters. The dynamic parameters of retroglossal airway were also not statistically significant in these patients either. The anterior-posterior diameter $(3.8 \pm 0.4 \mathrm{~mm}$ during awake and $3.9 \pm 0.6 \mathrm{~mm}$ during asleep) and cross sectional area of retroglossal airway (during $108.9 \pm 15 \mathrm{~mm}$ during awake and $113.5 \pm$ $26 \mathrm{~mm}$ during asleep) and the awaken and asleep states were also not statistically significant (Table 4)

So, we measured comprehensive airway dimensions such as anteroposterior diameter of the tongue, midline saggital diameter of tongue, intermandibular rami distance, mentum spine distance and relative size of the tongue using the spine as the posterior border of the bony confines. Among these parameters, only the relative size of the tongue (not the absolute size of the tongue ) was statistically significant (Table 5). The rest of the parameters, did not show any statistical significance. Since we did not employ any healthy controls in the study, we compared these parameters from significant retropalatal airway obstruction alone.

\section{(discussed later).}

Thus, it is well clear that, retropalatal airway is a significant cause of obstruction in patients with OSA in our part of the world, compared with retroglossal airway. The use MRI parameters, such as cross sectional area and antero-posterior diameter of the retropalatal airway during the respiratory phase in asleep and awakened states proves valuable as tool for objective measurement of OSA. However, when retroglossal airway is concerned, our study shows that the relative size of the tongue, offers more advantage than the variables of airway compromise such as cross sectional area and antero-posterior diameter. The use of these variables and dimensions as a tool for predicting the outcome of the cross sectional area-concerned surgeries such as, uveo-palato-plasty for retropalatal airway compromise, and genioglossus for retroglossal airway compromise, needs extensive follow up studies.

\section{DISCUSSION:}

The study was with this new focus in mind that we evaluated MR sleep studies for quantitative MR parameters to predict the airway narrowing in a way that would help patients with Obstructive Sleep Apnea(OSA) in deciding the management.

We found different types of upper airway obstruction, and we classified OSAS subjects into three groups: those with retropalatal obstruction only, those with retrolingual obstruction only and those with both retropalatal and retrolingual obstruction. However no patients had both retropalatal and retrolingual obstruction in our study. This classification is in par with Il Joon Moon et al. ${ }^{16}$, who primarily studied the retropalatal, retroglossal and combined airway to assess real-time obstruction sites and correlated the same with sleep parameters such as supine RDI and MinSaO2. According to them, subjects with both levels of obstruction showed higher supine RDI and lower Min- $\mathrm{SaO} 2$ than subjects without obstruction or with retropalatal obstruction only. It can be inferred that classifying the three groups according to sagittal images was a reliable and valid method because there were significant differences in various parameters between the groups. Our study not only classifies the airway based on subjective assessment of visible obstruction as performed by Il Joon Moon et al., but also provides objective measurements to substantiate the same.

According to Butorova et $\mathrm{al}^{17}$. who measured the diameter of the RP (region retropalatal) and $\mathrm{RG}$ (region retroglossal) area in control group and OSA group, and showed that there is significant difference in these groups. Our study showed significant differences in the mean minimum anterior-posterior diameter of retropalatal airway during respiratory cycle during awakened and sleep states ( $\mathrm{p}$-value 0.03 ). These dimensions are comparable with the RP (region retropalatal) diameter measured by Butorova et $\mathrm{al}^{17}$. Not only did we measure the diameter of the retropalatal airway as performed by Butorova et al.we also used cross sectional area of retroglossal and retropalatal airways during respiratory cycle, which is a better correlate. Our study showed significant differences in mean minimum cross sectional area of retropalatal airway during respiratory cycle during awakened and sleep states (p-value 0.04)

The diameters and the area of RG (region retroglossal) showed no significant differences, in our study, contrary to what is stated by Butorova et $\mathrm{al}^{17}$. in the awakened and sleep states of the OSA group (pvalue 0.43 ). This may be attributed to the racial and regional heterogeneity, since, the retropalatal airway is mostly affected in our part of the world, rather than retroglossal airway. 
According to Jose E. Barrera et $\mathrm{al}^{18}$, who measured Posterior Airway Space (PAS) as the airway cross-sectional area from the nasal plane to the mandibular plane (airspace between nasal and mandibular planes), showed significant difference in both nasal and mandibular PAS distance and PAS area between control and OSA group. Though we did not typically measure Posterior Airway Space (PAS) as mentioned by Jose E. Barrera et al, we measured maximum and minimum area of retroglossal and retropalatal airways separately during respiratory cycle, which is a close modification. Our study shows significant difference in the retropalatal airway during respiratory cycle during the awakened and sleep states. However, the dynamic parameters of retroglossal airway were not statistically significant.

The fact that relative but not absolute tongue size is a clinically important parameter is consistent with the results of previous studies in the literature. The importance of relative tongue size as opposed to absolute tongue size was also discussed by Guimaraes et $\mathrm{a}^{19}$. Only less than one third our patients had retroglossal airway compromise in our study.

Among them, the relative size of the tongue using the spine as the posterior border of the bony confines proved more valuable in establishing the diagnosis of the retroglossal airway compromise, than the area of retroglossal airway during respiratory cycle during awakened and sleep states. We used OSA cases with significant retropalatal obstruction alone as controls, to establish that the relative size of the tongue is a valuable indicator for retroglossal airway. However, this could influence the study in various ways.

According to Lisa M. Walter et al. who studied OSA in adults has been associated with injury to areas of the brain that have multiple functions and involve both gray and white matter. Areas impacted include the insular, cingulate, ventral medial prefrontal cortices, cerebella deep nuclei and cortex, anterior hypothalamus, raphé, ventrolateral medulla, and basal ganglia ${ }^{20}$. Furthermore, Lisa M. Walter et al. also observed that, adult OSA has been associated with significant axonal injury, notably in limbic structures related to affective processes; pontine projections to the cerebellum, which are essential motor and blood pressure regulatory fibers; and the cingulum bundle within the anterior cingulate cortex, which is important for respiratory patterning. Our study did not reveal any major structural abnormalities in brain, in patients with OSAS except for some white matter hyperintensities, which was attributed to the age related findings ${ }^{21-22}$. However, we did not employ any DTI (Diffusion Tensor Imaging) in our study group to rule out microstructural integregrity of the white matter tracts as performed by Lisa M. Walter et al. Outside the airway, nearly half our patients showed cervical disc degeneration, loss of cervical lordosis and prominent osteophytes. Weather these changes contribute to OSAS or not is still debatable.

Along with the various parameters, Ciscar et $\mathrm{al}^{23}$. studied the diemensions of the soft palate among the OSA group and the control group showed that there is significant differences between them. The area of the soft palate measured by Ciscar et al. in the control group was $322 \pm 23 \mathrm{~mm}$. Our study showed significant increase in the thickness of the soft palate $445 \pm 123 \mathrm{~mm}$ in the OSA group, compared with the control group employed by Ciscar et al. Therefore, we believe that in most of our cases, the decrease in the retropalatal airway dimensions were primarily attributed to the thickness of the soft palate.

According to William E. Schaaf et al. who studied the dimensions of the airways in predicting the outcome of the genioglossus advancement surgery in children, concluded that the larger size of the adenoid tonsils on MRI tonsils on MRI were predictors of failure of genioglossus advancement for the treatment of glossoptosis causing $\mathrm{OSA}^{24}$. Since, we did not include pediatric population in our study and a few of our OSA group (primarily adults) had larger size of adenoids, we could establish a significant correlation adenoid hypertrophy and OSA.

\section{LIMITATIONS:}

Limitations of this study include the moderate sample size of the OSA group, and we did not any control group. We included control groups from various studies, which would influence the study in a manner of racial heterogeneity.
OSA group (primarily adults) had larger size of adenoids, we could not establish a significant correlation adenoid hypertrophy and OSA.

We excluded patients with a body mass index (BMI) greater than 40 $\mathrm{kg} / \mathrm{m}^{2}$ and those with contraindications to MRI, due to constraints imposed by the bore of the magnet. We used OSA cases with significant retropalatal obstruction alone as controls, to establish that the relative size of the tongue is a valuable indicator for retroglossal airway. However, this could influence the study in various ways.

\section{CONCLUSION:}

Our study shows that retropalatal airway is a significant cause of obstruction in patients with OSA, compared with retroglossal airway. We suggest that, when the compromise of the retroplatal airway is to be evaluated, variables such as cross sectional area and antero-posterior diameter of the retropalatal airway during the respiratory phase in asleep and awakened states and diemensions such as thickness of the soft palate proves valuable, as a tool for objective measurement of OSA. When the compromise of the retroglossal airway is to be evaluated, the dimensions such as the relative size of the tongue, offers more advantage than the variables of airway compromise such as cross sectional area and antero-posterior diameter. To evaluate further if some of our parameters correlate with the outcomes of the surgeries of OSA, a multivariate analysis would have been required.

\section{REFERENCES:}

1. American Academy of Sleep Medicine. Obstructive sleep apnea syndrome. The International Classification of Sleep Disorders Revised Diagnostic and Coding Manual; 2001 .

2. Reddy EV, Kadhiravan T, Mishra HK, et al. Prevalence and risk factors of obstructive sleep apnea among middle-aged urban Indians: a community-based study. Sleep Med. 2009;10:913-8.

3. Udwadia ZF, Doshi AV, L onkar SG, Singh CI. Prevalence of sleep disordered breathing and sleep apnoea in middle-aged urban Indian men. Am J Respir Crit Care Med 2004 $169: 168-73$

4. Vijayan VK, Patial K. Prevalence of obstructive sleep apnoea syndrome in Delhi, India. Chest 2006: 130:92S

5. Riley R, Guilleminault C, Herran J, Powell N. Cephalometric analyses and flow-volume loops in obstructive sleep apnea patients. Sleep. 1983;6:303e311.

6. deBerry-Borowiecki B, Kukwa A, Blanks RH. Cephalometric analysis for diagnosis and treatment of obstructive sleep apnea. Laryngoscope. 1988;98:226e234.

7. Boyd JH, Petrof BJ, Hamid Q, et al. Upper airway muscle inflammation and Boyd JH, Petrof BJ, Hamid Q, et al . Upper airway muscle inflammation
denervation changes in obstructive sleep apnea.Am J Respir Crit Care Med $2004 ;$;.

8. Friberg D, Ansved T, Borg K, et al . Histological indications of a progressive snorers disease in an upper airway muscle. Am J Respir Crit Care Med 1998

9. 17.Moore .K.Philips A pracitical method for describing patterns of tongue base narrowing(modification of Fujita ) in awakw adult patinets with obstructive sleep apnoea

10. Sher AE, Schechtman KB, Piccirillo JF. The efficacy of surgical modifications of the upper airway in adults with obstructive sleep apnea syndrome. Sleep $1996 ; 19: 156-$ 77.

11. Kolhi P, Jarnstedt J, Sikio M, Viik J, Dastidar P, Peltomaki T, et al. A texture analysis method for MR images of airway dilator muscles: a feasibility study. Dento maxillo facial radiology. 2014; 43 (5):20130403.

12. Abramson Z, Susarla S, August M, Troulis M, Kaban L. Three-dimensional computed tomographic analysis of airway anatomy in patients with obstructive sleep apnea. Journal of oral and maxillofacial surgery: official journal of the American Association of Oral and Maxillofacial Surgeons. 2010;68(2):354-62.

13. Arens R, McDonough JM, Costarino AT, Mahboubi S, Tayag-Kier CE, Maislin G, et al. Magnetic resonance imaging of the upper airway structure of children with obstructive sleep apnea syndrome. American journal of respiratory and critical care medicine. 2001; 164(4):698-703.

14. Hong JS, Oh KM, Kim BR, Kim YJ, Park YH. Three-dimensional analysis of pharyngeal airway volume in adults with anterior position of the mandible. American journal of orthodontics and dentofacial orthopedics: official publication of the American Association of Orthodontists, its constituent societies, and the American Board of Orthodontics. 2011; 140(4):e161-9.

15. Tong YB, Udupa JK, Odhner D. MR image analysis of upper airway architecture in children with OSAS. Conference proceedings: SPIE 9417, Medical Imaging 2015: Biomedical Applications in Molecular, Structural, and Functional Imaging. 2015;9417: 94172J1-6.

16. Moon IJ, Han DH, Kim JW, et al. Sleep magnetic resonance imaging as a new diagnostic method in obstructive sleep apnea syndrome. Laryngoscope. 2010;120(12):2546-2554.

17. E. Butorova, M. Shariya, D. Ustyuzhanin; Moscow/RU Magnetic resonance imaging in assessment of airway soft tissues in patients with obesity and obstructive sleep apnea. 10.1594/ecr2014/C-0995.

18. Jose E. Barrera, Candace Y. Pau, Veronique-Isabelle Forest, Andrew B. Holbrook, Gerald R. Popelka et al. Anatomic measures of upper airway structures in obstructive sleep apnea. World Journal of Otorhinolaryngology-Head and Neck Surgery 2017; 3 $85 \mathrm{e} 9$

19. Guimaraes CVA, Donnelly LF, Shott SR, et al.Adolescence with Down syndrome have relative rather than absolute macroglossia. Pediatr Radiol 2008; 38:1062-1067.

20. Walter LM, C Horne RS. Obstructive sleep-disordered breathing in children: Impact on the developing brain. Pediatr Respirol Crit Care Med 2018;2:58-64.

21. Naulaers G, Meyns B, Miserez M, Leunens V, Van Huffel S, Casaer P, et al. Use of tissue oxygenation index and fractional tissue oxygen extraction as non-invasive parameters for cerebral oxygenation. A validation study in piglets. Neonatology 2007;92:120-6

22. Harper RM, Kumar R, Macey PM, Woo MA, Ogren JA. Affective brain areas and sleepdisordered breathing. Prog Brain Res 2014;209:275-93

23. Ciscar MA, Juan G, Martinez V et al. Magnetic Resonance Imaging in OSA patients and healthy subjects. EurRespir J. 2001;17:79-86.

24. Tahmasian M, Rosenzweig I, Eickhoff SB, Sepehry AA, Laird AR, Fox PT, et al Structural and functional neural adaptations in obstructive sleep apnea: An activation likelihood estimation meta-analysis. Neurosci Biobehav Rev 2016;65:142-56. 